\title{
Approximate Approach to Magnetic and Thermodynamic Properties of Mixed Spin (1/2-S) Chains with $\mathrm{AB}$ and $\mathrm{AB}_{2}$ Topology
}

\author{
R. PEŁKA ${ }^{a, *}$ AND D. PINKOWICZ ${ }^{b}$ \\ ${ }^{a}$ The Niewodniczański Institute of Nuclear Physics, Polish Academy of Sciences \\ E. Radzikowskiego 152, 31-342 Kraków, Poland \\ ${ }^{b}$ Faculty of Chemistry, Jagiellonian University, Ingardena 3, 30-060 Kraków, Poland
}

\begin{abstract}
Motivated by the rapid development in the synthesis of novel molecule-based magnets, we have investigated magnetic and thermodynamic properties of mixed spin $(s-S)$ exchange coupled chains displaying a simple linear $\mathrm{AB}$ or a knotted $\mathrm{AB}_{2}$ arrangement. Approximate approach for $s=1 / 2$ and $S \geq 5 / 2$, treating at an intermediate step spin $S$ operator as a commuting variable and using the transfer matrix technique, is used. Susceptibility, magnetization and heat capacity of both spin systems are evaluated numerically from the corresponding free energy for $S=5 / 2$. Uniform ferromagnetic and antiferromagnetic couplings are discussed. The procedure reproduces the right values of saturation magnetization and the entropy content of the systems, corroborating its correctness. $\chi T$ curves are shown to depend crucially on the $\mu_{\mathrm{B}} H / J$ ratio. For zero-field heat capacity a double-peak structure is revealed for the $\mathrm{AB}$ chain, whereas for the $\mathrm{AB}_{2}$ chain only one broad anomaly is observed.
\end{abstract}

PACS numbers: 75.10.Pq, 71.70.Gm, 75.30.Cr, 75.40.Cx

\section{Introduction}

It is well known that combining transition metal ions with organic complexes opens rich possibilities to obtain magnetic coordination polymers displaying a variety of magnetic behaviors such as ferro-, antiferro-, and ferrimagnetism, canted antiferromagnetism and spin glass [1]. A distinct class is formed by the compounds with chain-like arrangements of spin carriers. From the chemical point of view they can be subdivided into homometallic [2], bimetallic [3], and heterometallic [4] chain structures. Moreover, the metal-radical hybrid strategy has resulted in several heterospin chain compounds [5], of which some show a 1D ferrimagnetic behavior [6] modeled by alternating spin chains [7]. Beside the strictly linear structures with unit cells of AA or AB type, both inorganic [8] and organic [9] compounds displaying the quasi $1 \mathrm{D}$ structures with the $\mathrm{AB}_{2}$ or $\mathrm{AB}_{1} \mathrm{~B}_{2}$ unit cell topologies have been reported.

Theoretical studies of such one-dimensional spin structures have been successfully trying to keep pace with the rapid development of chemical synthesis. The seminal result by Seiden [7] providing an exact formula for the zero-field susceptibility of the quantum-classical (AB) spin alternating chain has triggered further theoretical work on chain-like structures. At the end of the eighties his work was generalized to account for arbitrary spin quantum numbers [10] or even, drawing from the same principles, a model was proposed where whole quantum subsystems alternate with classical spins [11]. The $\mathrm{AB}_{2}$ type

* corresponding author; e-mail: robert.pelka@ifj.edu.pl chains have also been extensively studied either through the Hubbard Hamiltonian [12], exact diagonalization or quantum Monte Carlo methods [13], exact analytical approach based on the generalized decoration-iteration map [14], the transfer matrix technique for Ising-like interactions [15], or field theory methods [16].

\section{Approach}

The approximate approach goes along the lines developed in [17] to calculate the susceptibility for a spin alternating chain (AB type). Later it was generalized to account for the local anisotropy [18] and used to simulate the magnetization for two molecular magnets based on octacyanotungstate and lanthanide ions. It is easy to reformulate it so that it could be applied for spin alternating chains with $\mathrm{AB}_{2}$ topology defined by the spin Hamiltonian

$$
\begin{aligned}
\hat{H} & =-J \sum_{i=1}^{N} \hat{s}_{i}\left(\hat{S}_{1 i}+\hat{S}_{2 i}+\hat{S}_{1 i+1}+\hat{S}_{2 i+1}\right) \\
& +\mu_{\mathrm{B}} H \sum_{i=1}^{N}\left[G\left(\hat{S}_{z 1 i}+\hat{S}_{z 2 i}\right)+g \hat{s}_{z i}\right],
\end{aligned}
$$

where $g$ and $G$ denote the Landé factors of the corresponding spins. The main difference is that the elements of transfer matrix $\boldsymbol{T}$ are taken between the eigenstates of operators $\hat{\Sigma}_{i}$ and $\hat{\Sigma}_{i+1}$ corresponding, respectively, to sums $\hat{S}_{1 i}+\hat{S}_{2 i}$ and $\hat{S}_{1 i+1}+\hat{S}_{2 i+1}$ of two successive couples of spins $S$. In the thermodynamic limit $(N \rightarrow \infty)$ the partition function and the corresponding free energy is given by the largest eigenvalue of $\boldsymbol{T}: Z \cong \lambda_{\max }^{\mathrm{N}}$, $F \cong-\beta^{-1} N \ln \lambda_{\max }$. The magnetization, susceptibility, 
heat capacity, and entropy were calculated as appropriate derivatives of the free energy. The procedure has been encoded in a Mathematica\%.0 notebook.

\section{Results}

We tested the procedure by comparing the magnetization obtained for noninteracting spins $(J=0)$ to the corresponding sum of the Brillouin functions for an array of spin numbers $S \geq 5 / 2$ and magnetic field values. In the case of the $\mathrm{AB}$ chain a perfect agreement was found [18]. For the $\mathrm{AB}_{2}$ chain the relative deviation is higher but does not exceed $6 \%$. The calculated field dependence of isothermal magnetization was found to reproduce correctly the saturation values.

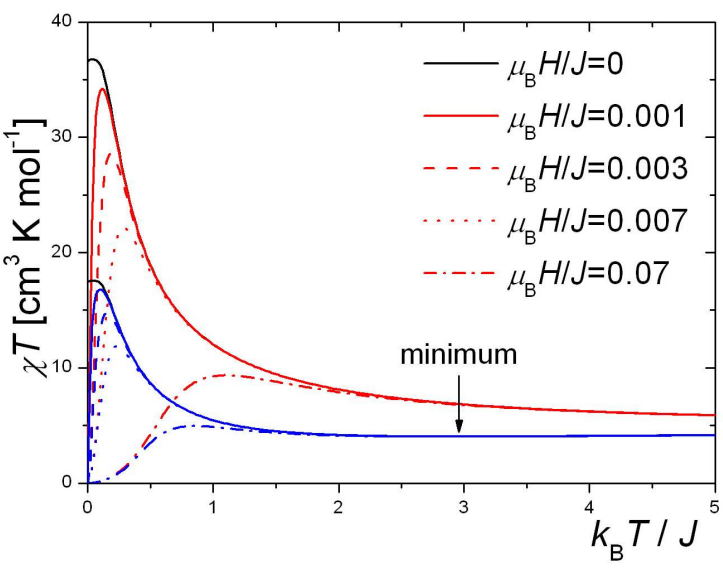

Fig. 1. $\chi T$ vs. $k_{\mathrm{B}} T / J$ for $\mathrm{AB}$ chain calculated with $G=g=2.0, S=5 / 2$, and an array of magnetic field values for ferromagnetic (red) and antiferromagnetic (blue) coupling. A minimum is observed for antiferromagnetic coupling at $k_{\mathrm{B}} T / J \approx 2.96$.

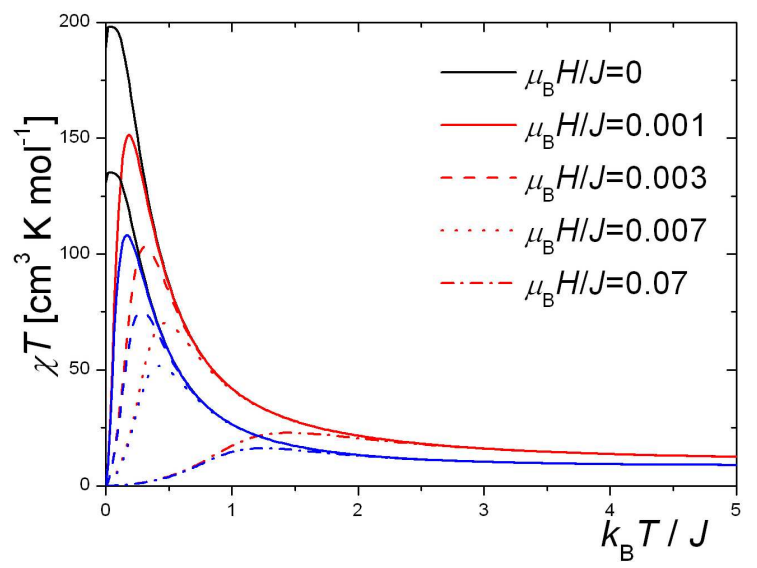

Fig. 2. $\chi T$ vs. $k_{\mathrm{B}} T / J$ for $\mathrm{AB}_{2}$ chain calculated with $G=g=2.0, S=5 / 2$, and an array of magnetic field values for ferromagnetic (red) and antiferromagnetic (blue) coupling.
Figures 1 and 2 show thermal dependence of $\chi T$ for some values of the external magnetic field, for $\mathrm{AB}$ and $\mathrm{AB}_{2}$ chain, respectively. For $\mathrm{AB}$ chain and antiferromagnetic coupling a minimum is observed at $k_{\mathrm{B}} T / J \approx 2.96$. This feature is absent for the $\mathrm{AB}_{2}$ chain.

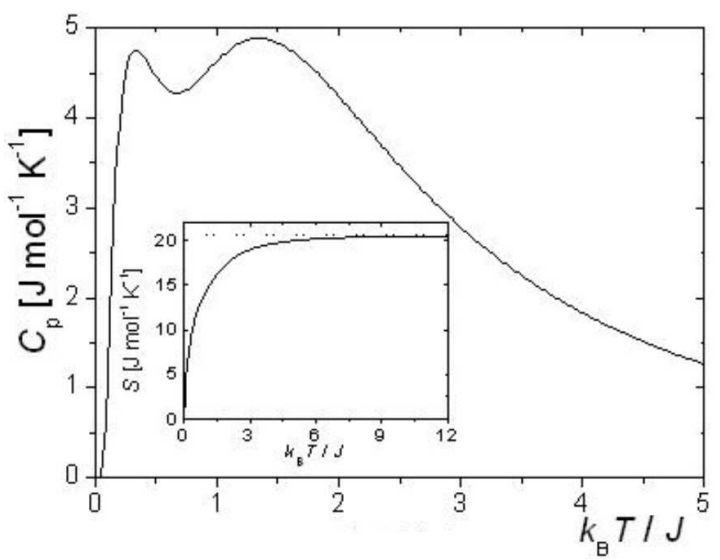

Fig. 3. Heat capacity vs. reduced temperature for $\mathrm{AB}$ chain calculated with $G=g=2.0, S=5 / 2$. Inset: temperature dependence of entropy saturating at the value of $R \ln (2(2 S+1)) \approx 20.7 \mathrm{~J} \mathrm{~mol}^{-1} \mathrm{~K}^{-1}$.

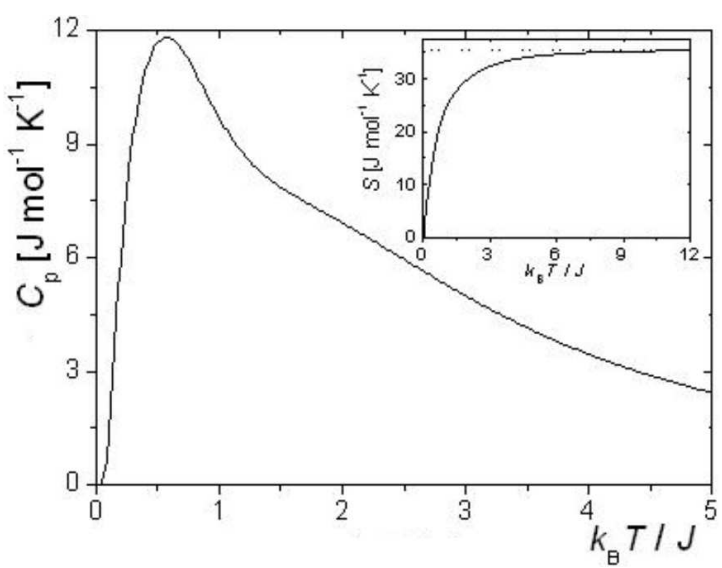

Fig. 4. Heat capacity vs. reduced temperature for $\mathrm{AB}_{2}$ chain calculated with $G=g=2.0, S=5 / 2$. Inset: temperature dependence of entropy saturating at the value of $R \ln \left(2(2 S+1)^{2}\right) \approx 35.6 \mathrm{~J} \mathrm{~mol}^{-1} \mathrm{~K}^{-1}$.

In Figs. 3 and 4 the temperature dependence of the zero-field heat capacity is shown for the $\mathrm{AB}$ and $\mathrm{AB}_{2}$ chains, respectively. For the $\mathrm{AB}$ chain a double peak anomaly with peaks at $k_{\mathrm{B}} T / J \approx 0.33$ and $k_{\mathrm{B}} T / J \approx$ 1.33 is observed, whereas for the $\mathrm{AB}_{2}$ chain one broad anomaly is present with the maximum at $k_{\mathrm{B}} T / J \approx 0.57$. The saturation values of entropy (see Insets of Figs. 3 and 4) corroborate the correctness of the approach. The approach provides an efficient means of calculating the magnetic and thermodynamic properties of the $\mathrm{AB}$ and $\mathrm{AB}_{2}$ chains. 


\section{Acknowledgments}

This work was partially supported by the Polish Ministry of Science and Higher Education within Research Project 0087/B/H03/2008/34.

\section{References}

[1] C.M. Wynn, M.A. Gîrtu, J.S. Miller, A.J. Epstein, Phys. Rev. B 56, 14050 (1997); C.M. Wynn, M.A. Gîrtu, J. Zhang, J.S. Miller, A.J. Epstein, Phys. Rev. B 58, 8508 (1998).

[2] M.S. Reis, A. Moreira dos Santos, V.S. Amaral, P. Brando, J. Rocha, Phys. Rev. B 73, 214415 (2006).

[3] S. Yamamoto, Phys. Rev. B 69, 064426 (2004).

[4] R. Clérac, H. Miyasaka, M. Yamashita, C. Coulon, J. Am. Chem. Soc. 124, 12837 (2002).

[5] A.S. Ovchinnikov, I.G. Bostrem, V.E. Sinitsyn, A.S. Boyarchenkov, N.V. Baranov, K. Inoue, J. Phys., Condens. Matter 14, 8067 (2002).

[6] K. Fegy, D. Luneau, E. Belorizky, M. Novac, J.-L. Tholence, C. Paulsen, T. Ohm, P. Rey, Inorg. Chem. 37, 4524 (1998).

[7] J. Seiden, J. Phys. Lett. 44, 947 (1983).

[8] M. Matsuda, K. Kakurai, A.A. Belik, M. Azuma, M. Takano, M. Fujita, Phys. Rev. B 71, 144411 (2005); H. Kikuchi, Y. Fujii, M. Chiba, S. Mitsudo, T. Idehara, T. Tonegawa, K. Okamoto, T. Sakai, T. Kuwai, H. Ohta, Phys. Rev. Lett. 94, 227201 (2005); H.H. Fu, K.L. Yao, Z.L. Liu, Phys. Rev. B 73, 104454 (2006); B. Gu, G. Su, Phys. Rev. B 75, 174437 (2007).

[9] Y. Hosokoshi, K. Katoh, Y. Nakazawa, H. Nakano, K. Inoue, J. Am. Chem. Soc. 123, 7921 (2001); K.L. Yao, Q.M. Liu, Z.L. Liu, Phys. Rev. B 70, 224430 (2004); K.L. Yao, H.H. Fu, Z.L. Liu, Solid State Commun. 135, 197 (2005).
[10] R. Georges, J. Curély, J.C. Gianduzzo, Q. Xu, O. Kahn, Y. Pei, Physica B 153, 77 (1988); J. Curély, Ph.D. Thesis, Université de Bordeaux I, 1990.

[11] J. Curély, R. Georges, Phys. Rev. B 46, 3520 (1992).

[12] M.D. Coutinho-Filho, R.R. Montenegro-Filho, E.P. Raposo, C. Vitoriano, M.H. Oliveira, J. Braz. Chem. Soc. 19, 232 (2008).

[13] S. Yamamoto, J. Ohara, Phys. Rev. B 76, 014409 (2007); N.B. Ivanov, Condens. Matter Phys. 12, 435 (2009).

[14] L. Čanová, J. Strečka, M. Jaščur, J. Phys., Condens. Matter 18, 4967 (2006).

[15] H.H. Fu, K.L. Yao, Z.L. Liu, J. Magn. Magn. Mater. 305, 253 (2006).

[16] E.P. Raposo, M.D. Coutinho-Filho, Phys. Rev. B 59, 14384 (1999).

[17] M. Verdaguer, A. Gleizes, J.P. Renard, J. Seiden, Phys. Rev. B 29, 5144 (1984).

[18] R. Pełka, M. Bałanda, P. Przychodzeń, K. Tomala, B. Sieklucka, T. Wasiutyński, Phys. Status Solidi $C$ 3, 216 (2006); P. Przychodzeń, K. Lewiński, R. Pełka, M. Bałanda, K. Tomala, B. Sieklucka, Dalton Trans., 625 (2006). 